\title{
Stability analysis for impulsive stochastic fuzzy $p$-Laplace dynamic equations under Neumann or Dirichlet boundary condition
}

Ruofeng Rao ${ }^{1 *}$ and Zhilin $\mathrm{Pu}^{1,2}$

"Correspondence:

ruofengrao@163.com

${ }^{1}$ Institution of Mathematics, Yibin

University, Yibin, Sichuan 644007, P.R. China

Full list of author information is

available at the end of the article

\begin{abstract}
Under Neumann or Dirichlet boundary conditions, the stability of a class of delayed impulsive Markovian jumping stochastic fuzzy $p$-Laplace partial differential equations (PDEs) is considered. Thanks to some methods different from those of previous literature, the difficulties brought by fuzzy stochastic mathematical model and impulsive model have been overcome. By way of the Lyapunov-Krasovskii functional, Itô formula, Dynkin formula and a differential inequality, new LMI-based global stochastic exponential stability criteria for the above-mentioned PDEs are established. Some applications of the obtained results improve some existing results on neural networks. And some numerical examples are presented to illustrate the effectiveness of the proposed method due to the significant improvement in the allowable upper bounds of time delays.
\end{abstract}

MSC: 34D20; 34D23; 34B45; 34B37; 34K20

Keywords: differential inequality; Laplace diffusion; Markovian jumping

\section{Introduction}

In this paper, we are concerned with the following delayed impulsive Markovian jumping stochastic fuzzy $p$-Laplace partial differential equations (PDEs):

$$
\left\{\begin{aligned}
d v_{i}(t, x)= & {\left[\sum_{k=1}^{m} \frac{\partial}{\partial x_{k}}\left(\mathcal{D}_{i k}(t, x, v)\left|\nabla v_{i}(t, x)\right|^{p-2} \frac{\partial v_{i}}{\partial x_{k}}\right)\right.} \\
& -\mathfrak{b}_{i}\left(v_{i}(t, x)\right)+\bigwedge_{j=1}^{n} \hat{c}_{i j}(r(t)) \mathfrak{F}_{j}\left(v_{j}(t, x)\right) \\
& +\bigvee_{j=1}^{n} \check{c}_{i j}(r(t)) \mathfrak{F}_{j}\left(v_{j}(t, x)\right)+\bigwedge_{j=1}^{n} \hat{d}_{i j}(r(t)) \mathfrak{G}_{j}\left(v_{j}\left(t-\tau_{j}(t), x\right)\right) \\
& \left.+\bigvee_{j=1}^{n} \check{d}_{i j}(r(t)) \mathfrak{G}_{j}\left(v_{j}\left(t-\tau_{j}(t), x\right)\right)\right] d t \\
& +\sum_{j=1}^{n} \sigma_{i j}\left(v_{j}(t, x), v_{j}\left(t-\tau_{j}(t)\right), x\right) d w_{j}(t), \\
& \text { for all } t \geq 0, t \neq t_{k}, x \in \Omega, \\
v\left(t^{+}, x\right)= & M_{k}(r(t)) v\left(t^{-}, x\right)+\mathcal{N}(r(t)) h\left(v\left(t^{-}-\tau(t), x\right)\right), \quad t=t_{k}, k=1,2, \ldots, \\
v(\theta, x)= & \phi(\theta, x), \quad(\theta, x) \in[-\tau, 0] \times \Omega,
\end{aligned}\right.
$$

equipped with the boundary condition

$$
\mathfrak{B}\left[v_{i}(t, x)\right]=0, \quad(t, x) \in[-\tau,+\infty) \times \partial \Omega, i=1,2, \ldots, n,
$$

O 2013 Rao and Pu; licensee Springer. This is an Open Access article distributed under the terms of the Creative Commons Attribution License (http://creativecommons.org/licenses/by/2.0), which permits unrestricted use, distribution, and reproduction in any medium, provided the original work is properly cited. 
where $p>1$ is a positive scalar, $\Omega \in R^{m}$ is a bounded domain with a smooth boundary $\partial \Omega$ of class $\mathcal{C}^{2}$ by $\Omega, v(t, x)=\left(v_{1}(t, x), v_{2}(t, x), \ldots, v_{n}(t, x)\right)^{T} \in R^{n}$. The smooth functions $\mathcal{D}_{j k}(t, x, v) \geq 0$. Denote $\tau(t)=\left(\tau_{1}(t), \tau_{2}(t), \ldots, \tau_{n}(t)\right)^{T}$, and $\tau_{j}(t)\left(0 \leq \tau_{j}(t) \leq \tau\right)$ corresponds to the transmission delays at time $t . v(t-\tau(t), x)=\left(v_{1}\left(t-\tau_{1}(t), x\right), v_{2}\left(t-\tau_{2}(t), x\right), \ldots, v_{n}(t-\right.$ $\left.\left.\tau_{n}(t), x\right)\right)^{T} \in R^{n} . t_{k}$ is called impulsive moment, satisfying $0<t_{1}<t_{2}<\cdots<t_{k}<\cdots$ with $\lim _{k \rightarrow \infty} t_{k}=\infty . v\left(t_{k}^{+}, x\right)$ and $v\left(t_{k}^{-}, x\right)$ denote the left-hand and right-hand limits at $t_{k}$, respectively. $h\left(v\left(t_{k}^{-}-\tau(t), x\right)\right)=\left(h_{1}\left(v_{1}\left(t_{k}^{-}-\tau_{1}\left(t_{k}\right)\right)\right), h_{2}\left(v_{2}\left(t_{k}^{-}-\tau_{2}\left(t_{k}\right)\right)\right), \ldots, h_{n}\left(v_{n}\left(t_{k}^{-}-\tau_{n}\left(t_{k}\right)\right)\right)\right)^{T}$, and $h_{j}\left(v_{j}\left(t_{k}^{-}-\tau_{j}\left(t_{k}\right)\right)\right)$ is the impulsive perturbation at time $t_{k}$. We always assume $v\left(t_{k}^{+}, x\right)=$ $v\left(t_{k}, x\right) . \mathfrak{b}_{j}\left(v_{j}(t, x)\right), \mathfrak{F}_{j}\left(v_{j}(t, x)\right)$ and $\mathfrak{G}_{j}\left(v_{j}(t, x)\right)$ are continuous functions. $\bigwedge$ and $\bigvee$ denote the fuzzy AND and OR operation, respectively. Each $w_{j}(t)$ is scalar standard Brownian motion defined on a complete probability space $(\boldsymbol{\Omega}, \mathcal{F}, \mathbb{P})$ with a natural filtration $\left\{\mathcal{F}_{t}\right\}_{t \geq 0}$. The noise perturbation $\sigma_{i j}: R \times R \rightarrow R$ is a Borel measurable function. $\{r(t), t \geq 0\}$ is a right-continuous Markov process on the probability space which takes values in the finite space $S=\{1,2, \ldots, N\}$ with generator $\Pi=\left\{\pi_{i j}\right\}$ given by

$$
\mathbb{P}(r(t+\delta)=j \mid r(t)=i)= \begin{cases}\pi_{i j} \delta+o(\delta), & j \neq i, \\ 1+\pi_{i j} \delta+o(\delta), & j=i,\end{cases}
$$

where $\pi_{i j} \geq 0$ is transition probability rate from $i$ to $j(j \neq i)$ and $\pi_{i i}=-\sum_{j=1, j \neq i}^{s} \pi_{i j}, \delta>0$ and $\lim _{\delta \rightarrow 0} o(\delta) / \delta=0$. In addition, the transition rates of the Markovian chain are considered to be partially available, namely, some elements in transition rates matrix $\Pi$ are time-invariant but unknown. For notational clarity, we denote $S=S_{k n}^{i} \cup S_{u n}^{i}$ with $S_{k n}^{i} \triangleq\left\{j\right.$, if $\pi_{i j}$ is known $\}$ and $S_{u n}^{i} \triangleq\left\{j\right.$, if $\pi_{i j}$ is unknown, and $\left.j \neq i\right\}$ for a given $i \in S$. $\varrho_{i}$ is a nonnegative scalar, satisfying $\varrho_{i} \geq \max _{j \in S_{u n}^{i}} \pi_{i j}$ for any given $i \in S$. In mode $r(t)=r \in S=$ $\{1,2, \ldots, N\}$, we denote $\hat{c}_{i j}(r(t))=\hat{c}_{i j}^{(r)}, \hat{d}_{i j}(r(t))=\hat{d}_{i j}^{(r)}, \check{c}_{i j}(r(t))=\check{c}_{i j}^{(r)}$ and $\check{d}_{i j}(r(t))=\check{d}_{i j}^{(r)}$. Besides, impulse parameters matrices $M_{k}(r(t))$ and $\mathcal{N}(r(t))$ are denoted by $M_{k r}$ and $\mathcal{N}_{r}$ for convenience. The boundary condition (1.1a) is called the Dirichlet boundary condition if $\mathfrak{B}\left[v_{i}(t, x)\right]=v_{i}(t, x)$, and the Neumann boundary condition if $\mathfrak{B}\left[v_{i}(t, x)\right]=\frac{\partial v_{i}(t, x)}{\partial \nu}$, where $\frac{\partial v_{i}(t, x)}{\partial \nu}=\left(\frac{\partial v_{i}(t, x)}{\partial x_{1}}, \frac{\partial v_{i}(t, x)}{\partial x_{2}}, \ldots, \frac{\partial v_{i}(t, x)}{\partial x_{m}}\right)^{T}$ denotes the outward normal derivative on $\partial \Omega$.

Remark 1.1 PDEs (1.1) own a wide range of physics and engineering backgrounds. They admit the following three Cohen-Grossberg neural networks (CGNNs) as their special cases.

$$
\begin{aligned}
& \left\{\begin{aligned}
d v(t, x)= & \left\{\nabla \cdot\left(\mathcal{D}(t, x, v) \circ \nabla_{p} v(t, x)\right)-A(v(t, x))[B(v(t, x))-C f(v(t, x))\right. \\
& -D g(v(t-\tau(t), x))]\} d t+\sigma(v(t, x), v(t-\tau(t))) d w(t) \\
& \text { for all } t \geq 0, t \neq t_{k}, x \in \Omega \\
v\left(t_{k}^{+}, x\right)= & M_{k} v\left(t_{k}^{-}, x\right)+\mathcal{N} h\left(v\left(t_{k}^{-}-\tau\left(t_{k}\right), x\right)\right), \quad k=1,2, \ldots \\
v(\theta, x)= & \phi(\theta, x), \quad(\theta, x) \in[-\tau(t), 0] \times \Omega,
\end{aligned}\right. \\
& \left\{\begin{aligned}
& \frac{\partial v(t, x)}{\partial t}= \mathcal{D} \Delta v(t, x)-A(v(t, x))[B(v(t, x))-C f(v(t, x))-D g(v(t-\tau(t), x))], \\
& \quad \text { for all } t \geq t_{0}, t \neq t_{k}, x \in \Omega, \\
& v\left(t_{k}^{+}, x\right)=M_{k} v\left(t_{k}^{-}, x\right)+\mathcal{N} h\left(v\left(t_{k}^{-}-\tau\left(t_{k}\right), x\right)\right), \quad k=1,2, \ldots, \\
& v(\theta, x)=\phi(\theta, x), \quad(\theta, x) \in[-\tau(t), 0] \times \Omega,
\end{aligned}\right.
\end{aligned}
$$




$$
\left\{\begin{aligned}
d x_{i}(t)= & \left\{-a_{i}\left(x_{i}(t)\right)\left[b_{i}\left(x_{i}(t)\right)-\bigwedge_{j=1}^{n} \hat{c}_{i j} f_{j}\left(x_{j}(t)\right)-\bigvee_{j=1}^{n} \check{c}_{i j} f_{j}\left(x_{j}(t)\right)\right.\right. \\
& \left.\left.-\bigwedge_{j=1}^{n} \hat{d}_{i j} g_{j}\left(x_{j}(t-\tau)\right)-\bigvee_{j=1}^{n} \check{d}_{i j} g_{j}\left(x_{j}(t-\tau)\right)\right]\right\} d t \\
& +\sum_{j=1}^{n} \sigma_{i j}\left(x_{j}(t), x_{j}(t-\tau)\right) d w_{j}(t), \\
x\left(t_{k}^{+}\right)= & M_{k} x\left(t_{k}^{-}\right)+\mathcal{N} h\left(v\left(t_{k}^{-}-\tau\left(t_{k}\right)\right)\right), \quad k=1,2, \ldots, \\
x_{i}(t)= & \phi_{i}(t), \quad-\tau \leq t \leq 0,
\end{aligned}\right.
$$

where $\mathcal{D}(t, x, v) \circ \nabla_{p} v(t, x)$ denotes the Hadamard product of matrix $\mathcal{D}(t, x, v)$ and $\nabla_{p} v$ (see, [1] or [2]), and $\mathcal{D}(t, x, v)=\left(\mathcal{D}_{j k}(t, x, v)\right)_{n \times m}$ satisfies $\mathcal{D}_{j k}(t, x, v) \geq 0$ for all $j, k,(t, x, v)$. $\sigma(v(t, x), v(t-\tau(t)))=\left(\sigma_{i j}\left(v_{j}(t, x), v_{j}\left(t-\tau_{j}(t)\right)\right)\right)_{n \times n}$, and $w(t)=\left(w_{1}(t), w_{2}(t), \ldots, w_{n}(t)\right)^{T}$. Throughout this paper, for the mode $r(t)=r \in S=\{1,2, \ldots, N\}$, we denote $C(r(t))=C_{r}=$ $\left(c_{i j}^{(r)}\right)_{n \times n}$, and $D(r(t))=D_{r}=\left(d_{i j}^{(r)}\right)_{n \times n} . \Delta v(t, x)=\left(\Delta v_{1}(t, x), \Delta v_{2}(t, x), \ldots, \Delta v_{n}(t, x)\right)^{T}$, and $\Delta v_{j}(t, x)=\sum_{k=1}^{m} \frac{\partial}{\partial x_{k}}\left(\frac{\partial v_{j}(t, x)}{\partial x_{k}}\right) \cdot x(t)=\left(x_{1}(t), x_{2}(t), \ldots, x_{n}(t)\right)^{T} \in R^{n}$.

The stability of $p$-Laplace diffusion stochastic CGNNs (1.2) was discussed by Xiongrui Wang, Ruofeng Rao and Shouming Zhong in 2012 [2], and the stability of deterministic system (1.3) was investigated by Xinhua Zhang, Shulin Wu and Kelin Li in 2011 [3]. Impulsive fuzzy CGNNs with nonlinear $p$-Laplace diffusion has never been studied as far as we know, and such a situation motivates our present study. Both the nonlinear $p$-Laplace diffusion and fuzzy mathematical model bring a great difficulty in setting up LMI criteria for the stability, and the stochastic functional differential equations model with nonlinear diffusion makes it harder. To study the stability of fuzzy CGNNs with diffusion, we have to construct a Lyapunov-Krasovskii functional in a non-matrix form (see, e.g., [4]). But stochastic mathematical formulae are always described in matrix forms. Furthermore, an impulsive model makes it harder. Recently, some new methods were employed to study the exponential stability for Markovian jumping, fuzzy neural networks in some related literature (see, e.g., [5-14]). Inspired by some methods and the idea of [3, 4] and the other above-mentioned papers, we overcame the difficulties brought by the Markovian jumping fuzzy impulsive model. By way of the Lyapunov-Krasovskii functional, Itô formula, Dynkin formula, the variational methods in Sobolev space $W^{1, p}(\Omega)$ (Lemma 2.1), and a differential inequality, new LMI-based global exponential stability criteria for the abovementioned PDEs are established; we obtain an LMI-based global stochastic exponential stability criterion of PDEs (1.1). Some applications to neural networks improve some existing results, which are illustrated by some numerical examples thanks to the significant improvement in the allowable upper bounds of time delays.

\section{Preliminaries}

Throughout this paper, we always assume that the following five conditions hold.

(H1) There exists a positive definite diagonal matrix $\mathscr{B}=\operatorname{diag}\left(\mathscr{B}_{1}, \mathscr{B}_{2}, \ldots, \mathscr{B}_{n}\right)$ such that

$$
\frac{\mathfrak{b}_{j}(r)}{r} \geq \mathscr{B}_{j}, \quad \forall j=1,2, \ldots, n \text {, and } 0 \neq r \in R \text {. }
$$

(H2) There exist positive definite diagonal matrices $F=\operatorname{diag}\left(F_{1}, F_{2}, \ldots, F_{n}\right)$, $G=\operatorname{diag}\left(G_{1}, G_{2}, \ldots, G_{n}\right)$ and $H=\operatorname{diag}\left(H_{1}, H_{2}, \ldots, H_{n}\right)$ such that

$$
\left|\mathfrak{F}_{j}\left(r_{1}\right)-\mathfrak{F}_{j}\left(r_{2}\right)\right| \leq F_{j}\left|r_{1}-r_{2}\right|, \quad\left|\mathfrak{G}_{j}\left(r_{1}\right)-\mathfrak{G}_{j}\left(r_{2}\right)\right| \leq G_{j}\left|r_{1}-r_{2}\right|,
$$




$$
\left|h_{j}\left(r_{1}\right)-h_{j}\left(r_{2}\right)\right| \leq H_{j}\left|r_{1}-r_{2}\right|
$$

for all $r_{1}, r_{2} \in R, j=1,2, \ldots, n$.

(H3) There exist nonnegative symmetric matrices $\mathcal{U}=\left(\mu_{i j}\right)_{n \times n}$ and $\mathcal{V}=\left(v_{i j}\right)_{n \times n}$ such that

$$
\operatorname{trace}\left[\sigma^{T}(u, v) \sigma(u, v)\right] \leq u^{T} u u+v^{T} \mathcal{V} v,
$$

where $u, v \in R^{n}, \sigma(u, v)=\left(\sigma_{i j}(u, v)\right)_{n \times n}$.

$(\mathrm{H} 4) \mathfrak{b}_{j}(0)=\mathfrak{F}_{j}(0)=\mathfrak{G}_{j}(0)=h_{j}(0)=0, \sigma_{i j}(0,0) \equiv 0, i, j=1,2, \ldots, n$.

It is obvious from (H4) that system (1.1) admits a zero solution $v(t, x ; 0) \equiv 0$ corresponding to the initial data $\phi=0$. For simplicity, we write $v(t, x ; \phi)=v(t, x)$. Denote $\mathbb{B}(v(t, x))=\left(\mathfrak{b}_{1}\left(v_{1}(t, x)\right), \mathfrak{b}_{2}\left(v_{2}(t, x)\right), \ldots, \mathfrak{b}_{n}\left(v_{n}(t, x)\right)\right)^{T}, \mathfrak{F}(v(t, x))=\left(\mathfrak{F}_{1}\left(v_{1}(t, x)\right), \mathfrak{F}_{2}\left(v_{2}(t, x)\right)\right.$, $\left.\ldots, \mathfrak{F}_{n}\left(v_{n}(t, x)\right)\right)^{T}, \mathfrak{G}(v(t, x))=\left(\mathfrak{G}_{1}\left(v_{1}(t, x)\right), \ldots, \mathfrak{G}_{n}\left(v_{n}(t, x)\right)\right)^{T}$. Denote $\sigma(t)=\sigma(v(t, x), v(t-$ $\tau(t), x))$ for short.

For convenience's sake, we introduce the following standard notations similar to those of [2].

$L^{2}(R \times \Omega), L_{\mathcal{F}_{0}}^{2}\left([-\tau, 0] \times \Omega ; R^{n}\right), Q=\left(q_{i j}\right)_{n \times n}>0(<0), Q=\left(q_{i j}\right)_{n \times n} \geq 0(\leq 0), Q_{1} \geq Q_{2}$ $\left(Q_{1} \leq Q_{2}\right), Q_{1}>Q_{2}\left(Q_{1}<Q_{2}\right), \lambda_{\max }(\Phi), \lambda_{\min }(\Phi)$, the identity matrix $I$ and the symmetric terms $*$.

In addition, we denote $|C|=\left(\left|c_{i j}\right|\right)_{n \times n}$ for any matrix $C=\left(c_{i j}\right)_{n \times n} ;|u(t, x)|=\left(\left|u_{1}(t, x)\right|\right.$, $\left.\left|u_{2}(t, x)\right|, \ldots,\left|u_{n}(t, x)\right|\right)^{T}$ for any $u(t, x)=\left(u_{1}(t, x), u_{2}(t, x), \ldots, u_{n}(t, x)\right)^{T}$.

Next, we give the following lemma, which is completely similar to [1, Lemma 2.3]. It can be derived by the Gauss formula (see, e.g., [2]).

Lemma 2.1 ([1, Lemma 2.3], [11, Lemma 6]) Let $P=\operatorname{diag}\left(p_{1}, p_{2}, \ldots, p_{n}\right)$ be a positive definite matrix, and let $v$ be a solution of system (1.1) with the boundary condition (1.1a). Then we have

$$
\begin{aligned}
\int_{\Omega} v^{T} P\left(\nabla \cdot\left(\mathcal{D}(t, x, v) \circ \nabla_{p} v\right)\right) d x \\
=-\sum_{k=1}^{m} \sum_{j=1}^{n} \int_{\Omega} p_{j} \mathcal{D}_{j k}(t, x, v)\left|\nabla v_{j}\right|^{p-2}\left(\frac{\partial v_{j}}{\partial x_{k}}\right)^{2} d x \\
=\int_{\Omega}\left(\nabla \cdot\left(\mathcal{D}(t, x, v) \circ \nabla_{p} v\right)\right)^{T} P v d x .
\end{aligned}
$$

\section{Main results}

Theorem 3.1 Assume that $p>1$. If the following three conditions hold:

(C1) there exist a sequence of positive scalars $\bar{\alpha}_{r}(r \in S)$ and positive definite diagonal matrices $P_{r}=\operatorname{diag}\left(p_{r 1}, p_{r 2}, \ldots, p_{r n}\right)(r \in S)$ such that the following LMI conditions hold:

$$
\begin{aligned}
& \Theta_{r}>0, \quad r \in S, \\
& P_{r}<\bar{\alpha}_{r} I, \quad r \in S,
\end{aligned}
$$


where matrices $\hat{C}_{r}=\left(\hat{c}_{i j}^{(r)}\right)_{n \times n}, \hat{D}_{r}=\left(\hat{d}_{i j}^{(r)}\right)_{n \times n}, \check{C}_{r}=\left(\check{c}_{i j}^{(r)}\right)_{n \times n}, \check{D}_{r}=\left(\check{d}_{i j}^{(r)}\right)_{n \times n}$, $\left|\hat{d}^{(r)}\right|=\max _{i, j}\left|\hat{d}_{i j}^{(r)}\right|, G_{0}=\max _{j} G_{j},\left|\check{d}^{(r)}\right|=\max _{i, j}\left|\check{d}_{i j}^{(r)}\right|$, and

$$
\begin{aligned}
& \Theta_{r}=-\left(\sum_{j \in S_{k n}^{r}} \pi_{r j} P_{j}+\varrho_{r} \sum_{j \in S_{u n}^{r}} P_{j}-2 P_{r} \mathcal{B}+P_{r}\left(\left|\hat{C}_{r}\right|+\left|\check{C}_{r}\right|\right) F+F\left(\left|\hat{C}_{r}^{T}\right|+\left|\check{C}_{r}^{T}\right|\right) P_{r}\right. \\
& \left.+n\left(\left|\hat{d}^{(r)}\right|+\left|\check{d}^{(r)}\right|\right) G_{0} P_{r}+\bar{\alpha}_{r} u\right) ;
\end{aligned}
$$

(C2) $a>b \geq 0$, where $a=\min _{r \in S}\left(\frac{\lambda_{\min } \Theta_{r}}{\lambda_{\max } P_{r}}\right), b=\max _{r \in S}\left(\frac{\lambda_{\max }\left(\bar{\alpha}_{r} \mathcal{v}+n\left(\left|\hat{d}^{(r)}\right|+\left|\check{d}^{(r)}\right|\right) G \lambda_{\max } P_{r}\right)}{\lambda_{\min } P_{r}}\right)$;

(C3) there exists a constant $\delta>1$ such that $\inf _{k \in Z}\left(t_{k}-t_{k-1}\right)>\delta \tau, \delta^{2} \tau>\ln \left(\rho e^{\lambda \tau}\right)$ and $\lambda-\frac{\ln \left(\rho e^{\lambda \tau}\right)}{\delta \tau}>0$, where $\rho=\max \left\{1, a_{j}+b_{j} e^{\lambda \tau}\right\}$ with $a_{j} \equiv \sup _{r, j}\left(\frac{2 \lambda_{\max }\left(\left|M_{r j}^{T}\right| P_{r}\left|M_{j r}\right|\right)}{\lambda_{\min } P_{r}}\right)$, $b_{j} \equiv \max _{r \in S}\left(\frac{2 \lambda_{\max }\left(H\left|\mathcal{N}_{r}^{T}\right| P_{r}\left|\mathcal{N}_{r}\right| H\right)}{\lambda_{\min } P_{r}}\right)$ for all $j \in \mathbb{Z}=\{1,2, \ldots\}$, and $\lambda>0$ is the unique solution of the equation $\lambda=a-b e^{\lambda \tau}$.

Then the null solution of impulsive Markovian jumping stochastic fuzzy system (1.1) is globally stochastically exponentially stable in the mean square with the convergence rate $\frac{1}{2}\left(\lambda-\frac{\ln \left(\rho e^{\lambda \tau}\right)}{\delta \tau}\right)$.

Proof Consider the Lyapunov-Krasovskii functional

$$
V(t, v(t), r)=\int_{\Omega} \sum_{i=1}^{n} p_{r i} v_{i}^{2}(t, x) d x, \quad \forall r \in S
$$

where $v(t, x)=\left(v_{1}(t, x), v_{2}(t, x), \ldots, v_{n}(t, x)\right)^{T}$ is a solution for stochastic fuzzy system (1.1). Sometimes we may denote $v(t, x)$ by $v, v_{i}(t, x)$ by $v_{i}$, and $\sigma(v(t, x), v(t-\tau(t), x))$ by $\sigma(t)$ for simplicity.

Let $\mathcal{L}$ be the weak infinitesimal operator. Then it follows by Lemma 2.1 that

$$
\begin{aligned}
\mathcal{L} V(t, v(t), r)= & -2 \sum_{k=1}^{m} \sum_{i=1}^{n} \int_{\Omega} p_{r i} \mathcal{D}_{i k}(t, x, v)\left|\nabla v_{i}\right|^{p-2}\left(\frac{\partial v_{i}}{\partial x_{k}}\right)^{2} d x \\
& -2 \sum_{i=1}^{n} \int_{\Omega} p_{r i} v_{i}\left[\mathfrak{b}_{i}\left(v_{i}\right)-\bigwedge_{j=1}^{n} \hat{c}_{i j}^{(r)} \mathfrak{F}_{j}\left(v_{j}\right)-\bigvee_{j=1}^{n} \check{c}_{i j}^{(r)} \mathfrak{F}_{j}\left(v_{j}\right)\right. \\
& \left.-\bigwedge_{j=1}^{n} \hat{d}_{i j}^{(r)} \mathfrak{G}_{j}\left(v_{j}(t-\tau(t), x)\right)-\bigvee_{j=1}^{n} \check{d}_{i j}^{(r)} \mathfrak{G}_{j}\left(v_{j}(t-\tau(t), x)\right)\right] d x \\
& +\int_{\Omega} v^{T} \sum_{j \in S} \pi_{r j} P_{j} v d x+\int_{\Omega} \operatorname{trace}\left(\sigma^{T}(t) P_{r} \sigma(t)\right) d x .
\end{aligned}
$$

On the other hand, we have

$$
\begin{aligned}
& 2 \sum_{i=1}^{n} p_{r i}\left|v_{i}\right| \sum_{j=1}^{n}\left|\hat{d}_{i j}^{(r)}\right| G_{j}\left|v_{j}(t-\tau(t), x)\right| \\
& \quad \leq v^{T}\left(n\left|\hat{d}^{(r)}\right| G_{0} P_{r}\right) v+v^{T}(t-\tau(t), x)\left(n\left|\hat{d}^{(r)}\right| \bar{\alpha}_{r} G\right) v(t-\tau(t), x),
\end{aligned}
$$




$$
\begin{aligned}
& 2 \sum_{i=1}^{n} p_{r i}\left|v_{i}\right| \sum_{j=1}^{n}\left|\check{d}_{i j}^{(r)}\right| G_{j}\left|v_{j}(t-\tau(t), x)\right| \\
& \quad \leq v^{T}\left(n\left|\check{d}^{(r)}\right| G_{0} P_{r}\right) v+v^{T}(t-\tau(t), x)\left(n\left|\check{d}^{(r)}\right| \bar{\alpha}_{r} G\right) v(t-\tau(t), x) .
\end{aligned}
$$

From $\pi_{r r}<0$ and the definition of $\varrho_{r}$, it is clear that $\sum_{j \in S} \pi_{r j} P_{j} \leq \sum_{j \in S_{k n}^{r}} \pi_{r j} P_{j}+$ $\varrho_{r} \sum_{j \in S_{u n}^{r}} P_{j}$.

So, we can conclude by $(\mathrm{H} 1)-(\mathrm{H} 4)$

$$
\begin{aligned}
\mathcal{L} V(t, v(t), r) \leq & \int_{\Omega}\left|v^{T}\right|\left(\sum_{j \in S_{k n}^{r}} \pi_{r j} P_{j}+\varrho_{r} \sum_{j \in S_{u n}^{r}} P_{j}-2 P_{r} B+2 P_{r}\left(\left|\hat{C}_{r}\right|+\left|\check{C}_{r}\right|\right) F\right. \\
& \left.+n\left(\left|\hat{d}^{(r)}\right|+\left|\check{d}^{(r)}\right|\right) G_{0} P_{r}+\bar{\alpha}_{r} u\right)|v| d x \\
& +\int_{\Omega} \bar{\alpha}_{r}\left[v^{T}(t-\tau(t), x)\left(\mathcal{V}+n\left(\left|\hat{d}^{(r)}\right|+\left|\check{d}^{(r)}\right|\right) G\right) v(t-\tau(t), x)\right] d x .
\end{aligned}
$$

Completely similar to (2.7)-(2.9) in [2], we can get by the Itô formula

$$
\begin{aligned}
D^{+} V(t, v(t), r) & \leq-\int_{\Omega}\left|v^{T}\right| \Theta_{r}|v| d x \\
& +\int_{\Omega} \bar{\alpha}_{r}\left[v^{T}(t-\tau(t), x)\left(\mathcal{V}+n\left(\left|\hat{d}^{(r)}\right|+\left|\check{d}^{(r)}\right|\right) G\right) v(t-\tau(t), x)\right] d x \\
& \leq-\min _{r \in S}\left(\frac{\lambda_{\min } \Theta_{r}}{\lambda_{\max } P_{r}}\right) V(t, v(t), r) \\
& +\max _{r \in S}\left(\frac{\lambda_{\max }\left(\bar{\alpha}_{r} \mathcal{V}+n\left(\left|\hat{d}^{(r)}\right|+\left|\check{d}^{(r)}\right|\right) G \lambda_{\max } P_{r}\right)}{\lambda_{\min } P_{r}}\right)[V(t, v(t), r)]_{\tau} .
\end{aligned}
$$

Owing to $V(t, v(t), r)=\int_{\Omega}\left|v^{T}\right| P_{r}|v| d x$, we can get

$$
\begin{aligned}
V\left(t_{k}, v\left(t_{k}\right), r\right)= & \int_{\Omega} v^{T}\left(t_{k}, x\right) P_{r} v\left(t_{k}, x\right) d x \\
= & \int_{\Omega}\left(M_{k r} v\left(t_{k}^{-}, x\right)+\mathcal{N}_{r} h\left(v\left(t_{k}^{-}-\tau\left(t_{k}\right), x\right)\right)\right)^{T} P_{r}\left(M_{k r} v\left(t_{k}^{-}, x\right)\right. \\
& \left.+\mathcal{N}_{r} h\left(v\left(t_{k}^{-}-\tau\left(t_{k}\right), x\right)\right)\right) d x \\
\leq & \frac{2 \lambda_{\max }\left(\left|M_{k r}^{T}\right| P_{r}\left|M_{k r}\right|\right)}{\lambda_{\min } P_{r}} V\left(t_{k}^{-}, v\left(t_{k}^{-}\right), r\right) \\
& +\frac{2 \lambda_{\max }\left(H\left|\mathcal{N}_{r}^{T}\right| P_{r}\left|\mathcal{N}_{r}\right| H\right)}{\lambda_{\min } P_{r}} \int_{\Omega}\left|v^{T}\left(t_{k}^{-}-\tau\left(t_{k}\right), x\right)\right| P_{r}\left|v\left(t_{k}^{-}-\tau\left(t_{k}\right), x\right)\right| d x \\
\leq & a_{k} V\left(t_{k}^{-}, v\left(t_{k}^{-}\right), r\right)+b_{k}\left[V\left(t_{k}^{-}, v\left(t_{k}^{-}\right), r\right)\right]_{\tau} .
\end{aligned}
$$

From (C3), it is not difficult to conclude that $\rho^{k+1} e^{k \lambda \tau} \leq e^{\left(\delta^{2}-\lambda\right) \tau} e^{\delta t_{k}}$, where $\lambda, a_{k}, b_{k}, \rho$ are defined in (C3), and so $\rho=\max _{k \in \mathbb{Z}}\left\{1, a_{k}+b_{k} e^{\lambda \tau}\right\}$. Then, by (C2), the differential inequality lemma ([2, Lemma 1.6]) yields $E V(t) \leq \rho[E V(0)]_{\tau} e^{-\left(\lambda-\frac{\ln \left(\rho e^{\lambda \tau}\right)}{\delta \tau}\right) t}$, or

$$
\left(\lambda_{\min } P_{r}\right) E\|\nu(t)\|_{2}^{2} \leq \rho\left(\lambda_{\max } P_{r} \sup _{-\tau \leq s \leq 0} E\|\phi(s)\|_{2}^{2}\right) e^{-\left(\lambda-\frac{\ln \left(\rho e^{\lambda \tau}\right)}{\delta \tau}\right) t},
$$


i.e.,

$$
E\|v(t)\|_{2}^{2} \leq \rho\left[\max _{r \in S}\left(\frac{\lambda_{\max } P_{r}}{\lambda_{\min } P_{r}}\right) \sup _{-\tau \leq s \leq 0} E\|\phi(s)\|_{2}^{2}\right] e^{-\left(\lambda-\frac{\ln \left(\rho \rho^{\lambda \tau}\right)}{\delta \tau}\right) t} .
$$

Therefore, we can see by the definition of global stochastic exponential stability (see, e.g., [15]) that the null solution of impulsive Markovian jumping stochastic fuzzy system (1.1) is globally stochastically exponentially stable in the mean square with the convergence rate $\frac{1}{2}\left(\lambda-\frac{\ln \left(\rho e^{\lambda \tau}\right)}{\delta \tau}\right)$.

Particularly for the case of $p=2$, we get from the Poincaré inequality (see, e.g., [16, Lemma 2.4]) that $\lambda_{1} \int_{\Omega}\left|v_{i}(t, x)\right|^{2} d x \leq \int_{\Omega}\left|\nabla v_{i}(t, x)\right|^{2} d x$, where $\lambda_{1}$ is the lowest positive eigenvalue of the boundary value problem

$$
\left\{\begin{array}{l}
-\Delta \varphi(t, x)=\lambda \varphi(t, x), \quad x \in \Omega, \\
\mathscr{B}[\varphi(t, x)]=0, \quad x \in \partial \Omega .
\end{array}\right.
$$

Theorem 3.2 Let $p=2$, and $\mathcal{D}_{0}=\inf _{i, k,(t, x v)} \mathcal{D}_{i k}(t, x, v)$. Then all the conclusions of Theorem 3.1 are true if its conditions are satisfied except that the $\Theta_{r}$ is replaced by $\Theta_{r}=$ $-\left(-2 \lambda_{1} \mathcal{D}_{0} P_{r}+\sum_{j \in S_{k n}^{r}} \pi_{r j} P_{j}+\varrho_{r} \sum_{j \in S_{u n}^{r}} P_{j}-2 P_{r} B+P_{r}\left(\left|\hat{C}_{r}\right|+\left|\check{C}_{r}\right|\right) F+F\left(\left|\hat{C}_{r}^{T}\right|+\left|\check{C}_{r}^{T}\right|\right) P_{r}+\right.$ $\left.n\left(\left|\hat{d}^{(r)}\right|+\left|\check{d}^{(r)}\right|\right) G_{0} P_{r}+\bar{\alpha}_{r} u\right)$.

Proof Indeed, if $p=2$, we can get by the Poincaré inequality

$$
\begin{aligned}
& -2 \sum_{k=1}^{m} \sum_{i=1}^{n} \int_{\Omega} p_{r i} \mathcal{D}_{i k}(t, x, v)\left|\nabla v_{i}\right|^{p-2}\left(\frac{\partial v_{i}}{\partial x_{k}}\right)^{2} d x \\
& =-2 \sum_{k=1}^{m} \sum_{i=1}^{n} \int_{\Omega} p_{r i} \mathcal{D}_{i k}(t, x, v)\left(\frac{\partial v_{i}}{\partial x_{k}}\right)^{2} d x \\
& \leq-2 \mathcal{D}_{0} \int_{\Omega} \sum_{i=1}^{n} p_{r i} \sum_{k=1}^{m}\left(\frac{\partial v_{i}}{\partial x_{k}}\right)^{2} d x \leq-2 \lambda_{1} \mathcal{D}_{0} \int_{\Omega} v^{T} P_{r} v d x .
\end{aligned}
$$

Then, by (3.3), we can similarly complete the rest of the proof by way of the methods in (3.4)-(3.10).

\section{Applications of main results in neural networks}

Let $\mathbb{B}(v(t, x))=A(v(t, x)) B(v(t, x))$ with $A(v(t, x))=\operatorname{diag}\left(a_{1}\left(v_{1}(t, x)\right), a_{2}\left(v_{2}(t, x)\right), \ldots, a_{n}\left(v_{n}(t\right.\right.$, $x))$ ) and $B(v(t, x))=\left(b_{1}\left(v_{1}(t, x)\right), b_{2}\left(v_{2}(t, x)\right), \ldots, b_{n}\left(v_{n}(t, x)\right)\right)^{T} \in R^{n}, \mathfrak{F}(v(t, x))=A(v(t, x)) \times$ $f(v(t, x))$, and $\mathfrak{G}(v(t, x))=A(v(t, x)) g(v(t, x))$ satisfy the following.

$\left(\mathrm{H} 1^{*}\right)$ There exist positive definite diagonal matrices $\underline{A}=\operatorname{diag}\left(\underline{a}_{1}, \underline{a}_{2}, \ldots, \underline{a}_{n}\right)$ and $\bar{A}=$ $\operatorname{diag}\left(\bar{a}_{1}, \bar{a}_{2}, \ldots, \bar{a}_{n}\right)$ such that

$$
0<\underline{a}_{i} \leq a_{i}(r) \leq \bar{a}_{i}
$$

for all $r \in R, i=1,2, \ldots, n$. 
$\left(\mathrm{H} 2^{*}\right)$ There exists a positive definite diagonal matrix $B=\operatorname{diag}\left(B_{1}, B_{2}, \ldots, B_{n}\right)$ such that

$$
\frac{b_{j}(r)}{r} \geq B_{j}, \quad \forall j=1,2, \ldots, n, \text { and } 0 \neq r \in R
$$

$\left(\mathrm{H} 3^{*}\right)$ There exist positive definite diagonal matrices $F=\operatorname{diag}\left(F_{1}, F_{2}, \ldots, F_{n}\right), G=\operatorname{diag}\left(G_{1}\right.$, $\left.G_{2}, \ldots, G_{n}\right)$ and $H=\operatorname{diag}\left(H_{1}, H_{2}, \ldots, H_{n}\right)$ such that

$$
\begin{aligned}
& \left|f_{j}\left(r_{1}\right)-f_{j}\left(r_{2}\right)\right| \leq F_{j}\left|r_{1}-r_{2}\right|, \quad\left|g_{j}\left(r_{1}\right)-g_{j}\left(r_{2}\right)\right| \leq G_{j}\left|r_{1}-r_{2}\right|, \\
& \left|h_{j}\left(r_{1}\right)-h_{j}\left(r_{2}\right)\right| \leq H_{j}\left|r_{1}-r_{2}\right|,
\end{aligned}
$$

for all $r_{1}, r_{2} \in R, j=1,2, \ldots, n$.

(H4*) There exist nonnegative symmetric matrices $\mathcal{U}=\left(\mu_{i j}\right)_{n \times n}$ and $\mathcal{V}=\left(v_{i j}\right)_{n \times n}$ such that

$$
\operatorname{trace}\left[\sigma^{T}(u, v) \sigma(u, v)\right] \leq u^{T} u u+v^{T} \mathcal{V} v
$$

where $u, v \in R^{n}, \sigma(u, v)=\left(\sigma_{i j}(u, v)\right)_{n \times n}$.

$\left(\mathrm{H} 5^{*}\right) b_{j}(0)=f_{j}(0)=g_{j}(0)=h_{j}(0)=0, \sigma_{i j}(0,0) \equiv 0, i, j=1,2, \ldots, n$.

Applying our main results to Cohen-Grossberg neural networks (CGNNs), we can conclude the following corollary from Theorem 3.1 directly.

\section{Corollary 4.1 If the following three conditions hold:}

(D1) there exist a positive scalar $\bar{\alpha}$ and a positive definite diagonal matrix $P$ such that the following LMI conditions hold:

$$
\begin{aligned}
& \Theta=-\left(-2 P \underline{A} B+P \bar{A}(|\hat{C}|+|\check{C}|) F+F\left(\left|\hat{C}^{T}\right|+\left|\check{C}^{T}\right|\right) \bar{A} P\right. \\
&\left.+n(|\hat{d}|+|\check{d}|) G_{0} P \bar{A}+\bar{\alpha} U\right)>0, \\
& P<\bar{\alpha} I,
\end{aligned}
$$

where matrices $\hat{C}=\left(\hat{c}_{i j}\right)_{n \times n}, \hat{D}=\left(\hat{d}_{i j}\right)_{n \times n}, \check{C}=\left(\check{c}_{i j}\right)_{n \times n}, \check{D}=\left(\check{d}_{i j}\right)_{n \times n},|\hat{d}|=\max _{i, j}\left|\hat{d}_{i j}\right|$, $\bar{A}_{0}=\max _{i} \bar{a}_{i}, G_{0}=\max _{j} G_{j},|\check{d}|=\max _{i, j}\left|\check{d}_{i j}\right|$;

(D2) $a>b \geq 0$, where $a=\frac{\lambda_{\min } \Theta}{\lambda_{\max } P}, b=\frac{\lambda_{\max }\left(\bar{\alpha} \mathcal{V}+n(|\hat{d}|+|\bar{d}|) \bar{A}_{0} G \lambda_{\max } P\right)}{\lambda_{\min } P}$;

(D3) there exists a constant $\delta>1$ such that $\inf _{k \in Z}\left(t_{k}-t_{k-1}\right)>\delta \tau, \delta^{2} \tau>\ln \left(\rho e^{\lambda \tau}\right)$ and $\lambda-\frac{\ln \left(\rho e^{\lambda \tau}\right)}{\delta \tau}>0$, where $\rho=\max \left\{1, a_{j}+b_{j} e^{\lambda \tau}\right\}$ with $a_{j} \equiv \sup _{j \in \mathbb{Z}}\left(\frac{2 \lambda_{\max }\left(\left|M_{j}^{T}\right| P\left|M_{j}\right|\right)}{\lambda_{\min } P}\right)$, $b_{j} \equiv \frac{2 \lambda_{\max }\left(H\left|\mathcal{N}^{T}\right| P|\mathcal{N}| H\right)}{\lambda_{\min } P}$ for all $j \in \mathbb{Z}=\{1,2, \ldots\}$, and $\lambda>0$ is the unique solution of the equation $\lambda=a-b e^{\lambda \tau}$.

Then the null solution of impulsive stochastic fuzzy system (1.4) is globally stochastically exponentially stable in the mean square with the convergence rate $\frac{1}{2}\left(\lambda-\frac{\ln \left(\rho e^{\lambda \tau}\right)}{\delta \tau}\right)$.

Remark 4.1 Corollary 4.1 not only extends [4, Theorem 3.1] from non-impulsive stochastic fuzzy CGNNs to impulsive stochastic fuzzy CGNNs, but also improves the criterion of [4, Theorem 3.1] from the non-matrix form to the more condensed matrix form, which can be efficiently tested and verified by computer Matlab LMI toolbox.

If Markovian jumping and fuzzy factors are ignored, we can conclude the following corollary. 
Corollary 4.2 Assume that $p>1$. If the following three conditions hold:

(E1) there exist a sequence of positive scalars $\bar{\alpha}$ and positive definite diagonal matrices $P$ such that the following LMI conditions hold:

$$
\begin{aligned}
& \widetilde{\Theta}>0, \\
& P<\bar{\alpha} I,
\end{aligned}
$$

where matrices $C=\left(c_{i j}\right)_{n \times n}, D=\left(d_{i j}\right)_{n \times n},|d|=\max _{i, j}\left|d_{i j}\right|, \bar{A}_{0}=\max _{i} \bar{a}_{i}$,

$G_{0}=\max _{j} G_{j}$, and $\widetilde{\Theta}=-\left(-2 P \underline{A} B+P \bar{A}|C| F+F\left|C^{T}\right| \bar{A} P+n|d| G_{0} P \bar{A}+\bar{\alpha} U\right) ;$

(E2) $a>b \geq 0$, where $a=\frac{\lambda_{\min } \widetilde{\Theta}}{\lambda_{\max } P}, b=\frac{\lambda_{\max }\left(\bar{\alpha} \mathcal{V}+\left.n|d|\right|_{0} G \lambda_{\max } P\right)}{\lambda_{\min } P}$;

(E3) there exists a constant $\delta>1$ such that $\inf _{k \in Z}\left(t_{k}-t_{k-1}\right)>\delta \tau, \delta^{2} \tau>\ln \left(\rho e^{\lambda \tau}\right)$ and

$\lambda-\frac{\ln \left(\rho e^{\lambda \tau}\right)}{\delta \tau}>0$, where $\rho=\max \left\{1, a_{j}+b_{j} e^{\lambda \tau}\right\}$ with $a_{j} \equiv \sup _{j \in \mathbb{Z}}\left(\frac{2 \lambda_{\max }\left(\left|M_{j}^{T}\right| P\left|M_{j}\right|\right)}{\lambda_{\min } P}\right)$,

$b_{j} \equiv \max _{r \in S}\left(\frac{2 \lambda_{\max }\left(H\left|\mathcal{N}^{T}\right| P|\mathcal{N}| H\right)}{\lambda_{\min } P}\right)$ for all $j \in \mathbb{Z}=\{1,2, \ldots\}$, and $\lambda>0$ is the unique

solution of the equation $\lambda=a-b e^{\lambda \tau}$,

then the null solution of impulsive stochastic system (1.2) is globally stochastically exponentially stable in the mean square with the convergence rate $\frac{1}{2}\left(\lambda-\frac{\ln \left(\rho e^{\lambda \tau}\right)}{\delta \tau}\right)$.

Remark 4.2 If letting $\mathcal{N}=0$, system (1.2) was investigated by [2]. However, LMIs criterion of Corollary 4.2 is more feasible and effective than that of [2, Theorem 2.1]. In fact, we know from the Schur complement theorem that the LMI condition of [2, Theorem 2.1] is equivalent to the inequality $\Upsilon=2 P_{1} \underline{A B}-P_{1} \bar{A}|C||C|^{T} \bar{A} P_{1}-P_{1} \bar{A}|D||D|^{T} \bar{A} P_{1}-F^{2}-P_{1} U-$ $P_{2}>0$, where the term $P \bar{A}|C|\left|C^{T}\right| \bar{A} P$ actually makes parameters amplify against $-\Upsilon<0$ if $\lambda_{\min } \bar{A}>1$ or $\lambda_{\min }|C|>1$. In other words, Corollary 4.2 can judge what [2, Theorem 2.1] cannot do, which may be illustrated by Example 5.2 (below).

Corollary 4.3 If the following three conditions hold:

(F1) there exist a positive scalar $\bar{\alpha}$ and a positive definite diagonal matrix $P$ such that the following LMI conditions hold:

$$
\bar{\Theta}>0,
$$

where matrices $C=\left(c_{i j}\right)_{n \times n}, D=\left(d_{i j}\right)_{n \times n},|d|=\max _{i, j}\left|d_{i j}\right|, \bar{A}_{0}=\max _{i} \bar{a}_{i}$, $G_{0}=\max _{j} G_{j}$, and $\bar{\Theta}=-\left(-2 \lambda_{1} P \mathcal{D}-2 P \underline{A} B+P \bar{A}|C| F+F\left|C^{T}\right| \bar{A} P+n|d| G_{0} P \bar{A}\right) ;$

(F2) $a>b \geq 0$, where $a=\frac{\lambda_{\min } \bar{\Theta}}{\lambda_{\max } P}, b=\frac{\lambda_{\max }\left(n|d| \overline{\overline{0}_{0}} G \lambda_{\max } P\right)}{\lambda_{\min } P}$;

(F3) there exists a constant $\delta>1$ such that $\inf _{k \in Z}\left(t_{k}-t_{k-1}\right)>\delta \tau, \delta^{2} \tau>\ln \left(\rho e^{\lambda \tau}\right)$ and $\lambda-\frac{\ln \left(\rho e^{\lambda \tau}\right)}{\delta \tau}>0$, where $\rho=\max \left\{1, a_{j}+b_{j} e^{\lambda \tau}\right\}$ with $a_{j} \equiv \sup _{j \in \mathbb{Z}}\left(\frac{2 \lambda_{\max }\left(\left|M_{j}^{T}\right| P\left|M_{j}\right|\right)}{\lambda_{\min } P}\right)$, $b_{j} \equiv \max _{r \in S}\left(\frac{2 \lambda_{\max }\left(H\left|\mathcal{N}^{T}\right| P|\mathcal{N}| H\right)}{\lambda_{\min } P}\right)$ for all $j \in \mathbb{Z}=\{1,2, \ldots\}$, and $\lambda>0$ is the unique solution of the equation $\lambda=a-b e^{\lambda \tau}$,

then the null solution of impulsive deterministic system (1.3) is globally stochastically exponentially stable in the mean square with the convergence rate $\frac{1}{2}\left(\lambda-\frac{\ln \left(\rho \rho^{\lambda \tau}\right)}{\delta \tau}\right)$.

Remark 4.3 For the same reason as in Remark 4.2, the LMI (4.5) of Corollary 4.3 is more feasible and effective than that of [3, Theorem 3.1], which may be illustrated by a numerical example below (Example 5.1). 


\section{Numerical examples}

In this section, two examples are given to illustrate that the criteria of Corollary 4.2 and Corollary 4.3 can judge what some existing criteria cannot do. The third numerical example is presented to illustrate the effectiveness of our main results (Theorems 3.1-3.2).

Example 5.1 Consider impulsive system (1.3) with the following parameters:

$$
\begin{array}{ll}
\underline{A}=\operatorname{diad}(0.1,0.1), \quad \bar{A}=\operatorname{diag}(0.2,0.2), \quad B=\operatorname{diag}(2,2), \\
F=\operatorname{diag}(0.1,0.2), \quad M=\operatorname{diag}(1.2,1.2) .
\end{array}
$$

In this section, we denote

$$
[a, b ; c, d]=\left(\begin{array}{ll}
a & b \\
c & d
\end{array}\right) .
$$

Assume, in addition, $G=\operatorname{diag}(1,1), \mathcal{D}=\operatorname{diag}(0.005,0.005), \mathcal{N}=\operatorname{diag}(0.0003,0.0003)$, $C=[3.2,-0.003 ;-0.003,3.2], D=[0.11,-0.003 ;-0.003,0.12], H=F, M_{k} \equiv M, \forall k=$ $1,2, \ldots v=\left(v_{1}(t, x), v_{2}(t, x)\right)^{T} \in R^{2}, x=\left(x_{1}, x_{2}\right)^{T} \in \Omega=\left\{\left(x_{1}, x_{2}\right)^{T} \in R^{2}:\left|x_{j}\right|<\sqrt{2}, j=1,2\right\}$. Assume that the boundary condition is the Dirichlet boundary one, and then $\lambda_{1}=\pi^{2}=$ 9.8696 (see, e.g., [16]). Assume that the lower limit of the time interval between impulses $\inf _{k \in Z}\left(t_{k}-t_{k-1}\right)=19$. From the differential inequality lemma [2, Lemma 1.6] we know that $\delta \tau<19$ and $\delta>1$, and hence the upper limit of time delay $\tau<19$.

With the above data, one can use computer Matlab LMI toolbox to solve the LMI (C1) of [3, Theorem 3.1], and obtain $t \min =-0.0516<0, P=\operatorname{diag}(0.5169,0.5147)$. Next, we need verify (C2) in [3, Theorem 3.1]. However, a direct computation derives $\tilde{a}=\frac{\lambda_{\min } \Theta}{\lambda_{\max } P}=\frac{0.0624}{0.5169}=$ $0.1207<0.1749=\frac{0.09}{0.5147}=\frac{\lambda_{\max }\left(G^{2}\right)}{\lambda_{\min } P}=b$, which implies $\widetilde{a}<\widetilde{b}$. Hence, [3, Theorem 3.1] cannot judge the stability of impulsive system (1.3) with the above data.

However, we can solve LMI (4.5) by Matlab LMI toolbox, and obtain $t \min =-0.5298$, $P=\operatorname{diag}(2.4462,2.3729)$. Further computation yields $a=\frac{\lambda_{\min } \bar{\Theta}}{\lambda_{\max } P}=\frac{0.5417}{2.4462}=0.2214, b=$ $\frac{\lambda_{\max }\left(n|d| \bar{A}_{0} G \lambda_{\max } P\right)}{\lambda_{\min } P}=\frac{0.0352}{2.3729}=0.0148$, and hence $a>b \geq 0$. In addition, a direct calculation derives $\rho=\max _{j}\left\{1, a_{j}+b_{j} e^{\lambda \tau}\right\}=2.9689$. Let $\delta=1.8093, \tau=10.5$, and then $\delta \tau=18.9977<$ $19=\inf _{k \in Z}\left(t_{k}-t_{k-1}\right), \lambda=0.1499, \delta^{2} \tau-\ln \left(\rho e^{\lambda \tau}\right)=31.7105>0$ and $\lambda-\frac{\ln \left(\rho e^{\lambda \tau}\right)}{\delta \tau}=0.0098>0$.

All the conditions (F1)-(F3) of Corollary 4.3 are satisfied, then by Corollary 4.3 the null solution of impulsive deterministic system (1.3) is globally stochastically exponentially stable in the mean square with the convergence rate 0.0049 and the allowable upper bound of time delays $\tau=10.5$.

Example 5.2 Under the Neumann boundary condition, we consider stochastic system (1.2) with the data (4.1) and the following parameters:

$$
\begin{aligned}
& G=\operatorname{diag}(0.3,0.3), \quad \mathcal{D}(t, v, x)=[0.005,0.003 ; 0.003,0.005], \\
& C=[2.2,-0.003 ;-0.003,2.2], \quad D=[0.11,-0.003 ;-0.003,0.12], \\
& \mathcal{U}=\operatorname{diag}(0.0003,0.0003), \quad \mathcal{V}=[0,0 ; 0,0]=\mathcal{N} .
\end{aligned}
$$

Let $\tau(t) \equiv \tau$, and then $\mu=0$. Now, one can use Matlab LMI toolbox to solve the LMI condition of [2, Theorem 2.1] and obtain $t \min =-0.0628<0, P_{1}=\operatorname{diag}(0.9084,0.9237)$, 
$P_{2}=\operatorname{diag}(0.0920,0.0778)$. Further computation yields that $\min \left\{\frac{\lambda_{\min } \widetilde{\Upsilon}}{\lambda_{\max } P_{1}},(1-\mu)\right\}=0.0832<$ $1.1568=\frac{\lambda_{\max } G^{2}}{\lambda_{\min } P_{2}}$, which implies that the condition $(\mathrm{C} 2)$ of [2, Theorem 2.1] is not satisfied. Hence, the stability of system (1.2) with the above data cannot be judged by [2, Theorem 2.1].

However, we solve LMIs (4.3)-(4.4), and obtain $t \min =-0.9343<0, \bar{\alpha}=42.5544$, $P=\operatorname{diag}(24.7366,23.9523)$. Moreover, we can get by direct computation that $a=0.1978>$ $0.0149=b$, and $\rho=2.9743$. Let $\tau=10.3, \delta=1.8445$ so that $\delta \tau=18.9979<19=\inf _{k \in Z}\left(t_{k}-\right.$ $\left.t_{k-1}\right), \delta^{2} \tau-\ln \left(\rho e^{\lambda \tau}\right)=32.5410>0$ and $\lambda-\frac{\ln \left(\rho e^{\lambda \tau}\right)}{\delta \tau}=0.0053>0$. All (E1)-(E3) of Corollary 4.2 are satisfied, then by Corollary 4.2 the null solution of impulsive stochastic system (1.2) is globally stochastically exponentially stable in the mean square with the convergence rate 0.0027 and the allowable upper bound of time delays $\tau=10.3$.

Example 5.3 Consider impulsive stochastic Markovian jumping fuzzy system (1.1) with the following parameters:

$$
\begin{aligned}
& \mathcal{D}(t, x, v)=[0.003,0.005 ; 0.004,0.006], \quad \mathscr{B}=\operatorname{diag}(2,2), \quad F=\operatorname{diag}(0.1,0.2), \\
& H=G=F, \quad U=\operatorname{diag}(0.0003,0.0003)=\mathcal{V}=\mathcal{N}_{r}, \\
& M_{k r} \equiv \operatorname{diag}(1.2,1.2), \quad \forall r \in S=\{1,2,3\}, k=1,2, \ldots
\end{aligned}
$$

In addition, $\hat{C}_{1}=[0.11,-0.003 ;-0.003,0.12]=\hat{D}_{1}, \check{C}_{1}=[0.16,-0.003 ;-0.003,0.18]=$ $\check{D}_{1}, \hat{C}_{2}=[0.13,-0.003 ;-0.003,0.15]=\hat{D}_{2}, \check{C}_{2}=[0.17,-0.003 ;-0.003,0.19]=\check{D}_{2}, \hat{C}_{3}=$ $[0.12,-0.003 ;-0.003,0.13]=\hat{D}_{3}, \breve{C}_{3}=[0.175,-0.003 ;-0.003,0.196]=\check{D}_{3}$.

The two cases of the transition rates matrices are considered as follows:

$$
\text { Case (1): } \Pi=\left(\begin{array}{ccc}
-0.2 & 0.1 & 0.1 \\
0.2 & -0.3 & 0.1 \\
0.3 & 0.1 & -0.4
\end{array}\right), \quad \text { Case (2): } \Pi=\left(\begin{array}{ccc}
-0.2 & ? & ? \\
0.2 & -0.3 & 0.1 \\
0.3 & 0.1 & -0.4
\end{array}\right) \text {, }
$$

$v=\left(v_{1}(t, x), v_{2}(t, x)\right)^{T} \in R^{2}, x=\left(x_{1}, x_{2}\right)^{T} \in \Omega=\left\{\left(x_{1}, x_{2}\right)^{T} \in R^{2}:\left|x_{j}\right|<\sqrt{2}, j=1,2\right\}$. Assume that the boundary condition is the Dirichlet boundary one, and then $\lambda_{1}=\pi^{2}=9.8696$. Assume that the lower limit of the time interval between pulses inf $\operatorname{sez}_{k \in Z}\left(t_{k}-t_{k-1}\right)=19$. From the differential inequality lemma (see, [17] or [2, Lemma 1.3]), we know that $\delta \tau<19$ and $\delta>1$, and hence the upper limit of time delay $\tau<19$ (see, Remark 5.1).

Now one can use Matlab LMI toolbox to solve the LMI conditions (3.1)-(3.2) of Theorem 3.1 for Case (1) and $p>1$, and obtain $t \min =-0.5607<0$, and $\alpha_{1}=25.4531$, $\alpha_{2}=25.4421, \alpha_{3}=25.4273, P_{1}=\operatorname{diag}(6.6617,6.7783), P_{2}=\operatorname{diag}(6.6320,6.7643), P_{3}=$ $\operatorname{diag}(6.6066,6.7304)$.

Moreover, a direct computation derives $a=3.7256, b=0.1399$. So, the condition (C2) $a>b \geq 0$ holds in this case.

By the definition of $a_{j}, b_{j}$, further computation derives $\rho=\max _{j}\left\{1, a_{j}+b_{j} e^{\lambda \tau}\right\}=2.9375$. Let $\tau=14$ and $\delta=1.3569$. Then solving the equation $\lambda=a-b e^{\lambda \tau}$ yields $\lambda=0.2299$. By these data, one can calculate that $\delta \tau=18.9972<19=\inf _{k \in Z}\left(t_{k}-t_{k-1}\right), \delta^{2} \tau-\ln \left(\rho e^{\lambda \tau}\right)=$ $21.4822>0$ and $\lambda-\frac{\ln \left(\rho e^{\lambda \tau}\right)}{\delta \tau}=0.0037>0$, which implies the condition (C3) of Theorem 3.1 holds. By Theorem 3.1, the null solution of impulsive Markovian jumping stochastic fuzzy system (1.1) is globally stochastically exponentially stable in the mean square with the convergence rate 0.00185 and the allowable upper bounds of time delays $\tau=14$. 
Assume that the boundary condition is the Dirichlet boundary one, and then $\lambda_{1}=$ $\pi^{2}=9.8696$ (see, e.g., [16]). Similarly, we can solve the corresponding conditions of Theorem 3.2 for Case (1) and $p=2$, and obtain $t \min =-0.5580<0, \bar{\alpha}_{1}=25.3516$, $\bar{\alpha}_{2}=25.3408, \bar{\alpha}_{3}=25.3262$, and $P_{1}=\operatorname{diag}(6.5896,6.7043), P_{2}=\operatorname{diag}(6.5604,6.6905)$, $P_{3}=\operatorname{diag}(6.5354,6.6572)$.

Similarly, we can calculate and obtain $a=3.7294, b=0.1399, \rho=2.9371$.

Let $\delta=1.3473$ and $\tau=14.1$, and then $\lambda=0.2284, \delta \tau=18.9972<19, \delta^{2} \tau-\ln \left(\rho e^{\lambda \tau}\right)=$ $21.2557>0$ and $\lambda-\frac{\ln \left(\rho e^{\lambda \tau}\right)}{\delta \tau}=0.0029>0$. By Theorem 3.2, the null solution of impulsive Markovian jumping stochastic fuzzy system (1.1) with $p=2$ is stochastically exponentially stable in the mean square with the convergence rate 0.00145 and the allowable upper bounds of time delays $\tau=14.1$.

Obviously in Case (2) we can assume $\varrho_{1}=0.2$. Next, we employ Matlab LMI toolbox to solve LMI conditions (3.1)-(3.2) of Theorem 3.1 for Case (2) and $p>1$, and obtain $t \min =-0.3196<0, \bar{\alpha}_{1}=25.7897, \bar{\alpha}_{2}=25.6385, \bar{\alpha}_{3}=25.6264, P_{1}=\operatorname{diag}(7.0171,7.1446)$, $P_{2}=\operatorname{diag}(6.7114,6.8458), P_{3}=\operatorname{diag}(6.6913,6.8174)$. So, $a=3.5752, b=0.1399, \rho=2.9377$.

Let $\delta=1.4072$ and $\tau=13.5$, and then $\lambda=0.2350, \delta \tau=18.9973<19, \delta^{2} \tau-\ln \left(\rho e^{\lambda \tau}\right)=$ $22.4827>0$ and $\lambda-\frac{\ln \left(\rho e^{\lambda \tau}\right)}{\delta \tau}=0.0113>0$. By Theorem 3.1, the null solution of impulsive Markovian jumping stochastic fuzzy system (1.1) is stochastically exponentially stable in the mean square with the convergence rate 0.00565 and the allowable upper bounds of time delays $\tau=13.5$.

Similarly, we can solve the corresponding conditions of Theorem 3.2 for Case (1) and $p=2$, and obtain $t$ min $=-0.5614<0, \bar{\alpha}_{1}=25.5625, \bar{\alpha}_{2}=25.4289, \bar{\alpha}_{3}=25.4165$, and $P_{1}=$ $\operatorname{diag}(6.8412,6.9638), P_{2}=(6.5701,6.7003), P_{3}=(6.5494,6.6714)$, and then $a=3.5777, b=$ $0.1399, \rho=2.9371$. Let $\delta=1.3969$ and $\tau=13.6$, and then $\lambda=0.0845$.

Further computation yields $\delta \tau=18.9973<19, \delta^{2} \tau-\ln \left(\rho e^{\lambda \tau}\right)=22.2850>0$ and $\lambda-$ $\frac{\ln \left(\rho e^{\lambda \tau}\right)}{\delta \tau}=0.0096>0$. By Theorem 3.2, the null solution of impulsive Markovian jumping stochastic fuzzy system (1.1) with $p=2$ is stochastically exponentially stable in the mean square with the convergence rate 0.0048 and the allowable upper bounds of time delays $\tau=13.6$.

Table 1 shows that the upper bounds of time delay decrease when there exist unknown elements of a transition rates matrix. This means that unknown elements of transition rates bring a great difficulty in judging the stability.

In some related literature [18, 19], their impulsive assumption is $M_{k}^{T} P M_{k}<P$. However, our impulse matrix $M_{k}$ may not satisfy the assumption of decreasing impulse. In all the above numerical examples, impulsive parameters matrices $M_{k}$ satisfy $\lambda_{\min } M_{k}=1.2>1$ so that $M_{k}^{T} P M_{k}>P$. Thereby, the increasing impulse not only brings some unstable factors to CGNNs, but also limits the time-delays' upper limit $\tau<\inf _{k \in Z}\left(t_{k}-t_{k-1}\right)$ (see [17] or [2, Lemma 1.6]).

Table 1 Allowable upper bounds of time delays and the convergence rate

\begin{tabular}{llllll}
\hline & \multicolumn{2}{l}{ Theorem $\mathbf{3 . 1}(\boldsymbol{p}>\mathbf{1})$} & & \multicolumn{2}{l}{ Theorem $\mathbf{3 . 2}(\boldsymbol{p}=\mathbf{2})$} \\
\cline { 2 - 3 } & Case (1) & Case (2) & & Case (1) & Case (2) \\
\hline Upper bound $\tau$ & 14 & 13.5 & & 14.1 & 13.6 \\
Convergence rate & 0.00185 & 0.00565 & & 0.00145 & 0.0048 \\
\hline
\end{tabular}


Remark 5.1 The parameters of impulsive deterministic system (1.3) do not satisfy the conditions of [3, Theorem 3.1] so that we are not sure whether system (1.3) is stable, for the conditions of [3, Theorem 3.1] are only sufficient ones, not necessary for the stability of system (1.3). However, we can conclude the stability by our Corollary 4.3, which implies that Corollary 4.3 allows for more effectiveness and less conservatism than [3, Theorem 3.1]. By the same token as in Remark 5.2, Corollary 4.2 is better than [2, Theorem 2.1].

Remark 5.2 Table 1 shows that the diffusion plays a positive role in the criterion of Theorem 3.2, which admits a wider range of time delays. Table 1 also illustrates the effectiveness and less conservatism of Theorems 3.1-3.2 due to the significant improvement in the allowable upper bounds of time delays.

Remark 5.3 Finding a solution $x$ to the LMI system $A(x)<B(x)$ is called the feasibility problem. So, in Examples 5.1-5.3, the system is feasible if $t \min <0$, and infeasible if $t$ min $>0$ (see [11, Remark 29(3)] for detail).

Competing interests

The authors declare that they have no competing interests.

Authors' contributions

All authors typed, read and approved the final manuscript.

\section{Author details}

${ }^{1}$ Institution of Mathematics, Yibin University, Yibin, Sichuan 644007, P.R. China. ${ }^{2}$ College of Mathematics and Software Science, Sichuan Normal University, Chengdu, Sichuan 610066, P.R. China.

\section{Acknowledgements}

The authors would like to thank the referees for their valuable suggestions. This work was supported by the Scientific Research Fund of Science Technology Department of Sichuan Province (2012JY010), and the Scientific Research Fund of Sichuan Provincial Education Department (12ZB349).

Received: 3 March 2013 Accepted: 4 May 2013 Published: 20 May 2013

\section{References}

1. Rao, R, Zhong, S, Wang, X: Stochastic stability criteria with LMI conditions for Markovian jumping impulsive BAM neural networks with mode-dependent time-varying delays and nonlinear reaction-diffusion. Commun. Nonlinear Sci. Numer. Simul. (accepted on May 18, 2013)

2. Wang, X, Rao, R, Zhong, S: LMI approach to stability analysis of Cohen-Grossberg neural networks with $p$-Laplace diffusion. J. Appl. Math. 2012, Article ID 523812 (2012)

3. Zhang, X, Wu, S, Li, K: Delay-dependent exponential stability for impulsive Cohen-Grossberg neural networks with time-varying delays and reaction-diffusion terms. Commun. Nonlinear Sci. Numer. Simul. 16, 1524-1532 (2011)

4. Zhu, Q, Li, X: Exponential and almost sure exponential stability of stochastic fuzzy delayed Cohen-Grossberg neural networks. Fuzzy Sets Syst. 203, 74-94 (2012)

5. Li, D, He, D, Xu, D: Mean square exponential stability of impulsive stochastic reaction-diffusion Cohen-Grossberg neural networks with delays. Math. Comput. Simul. 82(8), 1531-1543 (2012)

6. Li, B, Xu, D: Existence and exponential stability of periodic solution for impulsive Cohen-Grossberg neural networks with time-varying delays. Appl. Math. Comput. 219(5), 2506-2520 (2012)

7. Long, S, Xu, D: Stability analysis of stochastic fuzzy cellular neural networks with time-varying delays. Neurocomputing 74(14-15), 2385-2391 (2011)

8. Li, B, Xu, D: Mean square asymptotic behavior of stochastic neural networks with infinitely distributed delays. Neurocomputing 72(13-15), 3311-3317 (2009)

9. Tian, J, Li, Y, Zhao, J, Zhong, S: Delay-dependent stochastic stability criteria for Markovian jumping neural networks with mode-dependent time-varying delays and partially known transition rates. Appl. Math. Comput. 218(9), 5769-5781 (2012)

10. Ding, Y, Zhu, H, Zhong, S, Zeng, Y: Exponential mean-square stability of time-delay singular systems with Markovian switching and nonlinear perturbations. Appl. Math. Comput. 219(4), 2350-2359 (2012)

11. Rao, R, Wang, X, Zhong, S, Pu, Z: LMI approach to exponential stability and almost sure exponential stability for stochastic fuzzy Markovian-jumping Cohen-Grossberg neural networks with nonlinear $p$-Laplace diffusion. J. Appl. Math. 2013, Article ID 396903 (2013) 
12. Cheng, J, Zhu, H, Zhong, S, Li, G: Novel delay-dependent robust stability criteria for neutral systems with mixed time-varying delays and nonlinear perturbations. Appl. Math. Comput. 219(14), 7741-7753 (2013)

13. Zhao, H, Zhang, Q: Global impulsive exponential anti-synchronization of delayed chaotic neural networks. Neurocomputing 74(4), 563-567 (2011)

14. Zhao, H, Mao, Z: Boundedness and stability of nonautonomous cellular neural networks with reaction-diffusion terms. Math. Comput. Simul. 79(5), 1603-1617 (2009)

15. Zhu, Q, Li, X, Yang, X: Exponential stability for stochastic reaction-diffusion BAM neural networks with time-varying and distributed delays. Appl. Math. Comput. 217(13), 6078-6091 (2011)

16. Pan, J, Zhong, S: Dynamic analysis of stochastic reaction-diffusion Cohen-Grossberg neural networks with delays. Adv. Differ. Equ. 2009, Article ID 410823 (2009)

17. Yue, D, Xu, S, Liu, Y: Differential inequality with delay and impulse and its applications to design robust control. Control Theory Appl. 16(4), 519-524 (1999)

18. Rakkiyappan, R, Balasubramaniam, P: Dynamic analysis of Markovian jumping impulsive stochastic Cohen-Grossberg neural networks with discrete interval and distributed time-varying delays. Nonlinear Anal. Hybrid Syst. 3(4), 408-417 (2009)

19. Dong, M, Zhang, H, Wang, Y: Dynamics analysis of impulsive stochastic Cohen-Grossberg neural networks with Markovian jumping and mixed time delays. Neurocomputing 72(7-9), 1999-2004 (2009)

doi:10.1186/1687-2770-2013-133

Cite this article as: Rao and Pu: Stability analysis for impulsive stochastic fuzzy $p$-Laplace dynamic equations under Neumann or Dirichlet boundary condition. Boundary Value Problems 2013 2013:133.

\section{Submit your manuscript to a SpringerOpen ${ }^{\circ}$ journal and benefit from:}

- Convenient online submission

- Rigorous peer review

- Immediate publication on acceptance

Open access: articles freely available online

- High visibility within the field

- Retaining the copyright to your article 